\title{
La motricidad en el triatlón: otra mirada desde la física hacia la biomecánica*
}

Ana Margarita Torres-Aguila ${ }^{* *}$

\author{
Recibido: o9 de diciembre de 2020 \\ Aceptado: 17 de abril de 2021
}

Citar como:

Torres-Aguila, A. M. (2020). La motricidad en el triatlón: otra mirada desde la física hacia la biomecánica. Revista de Investigación Cuerpo, Cultura y Movimiento, 11(2). https://doi.org/10.15332/2422474X.6757

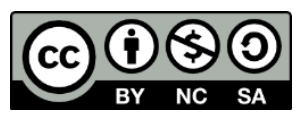

\section{Resumen}

Este artículo aborda aspectos de la motricidad, que se sustentan en los fundamentos de la biomecánica, en el razonamiento físico y el interdisciplinario. Estas ciencias en el preuniversitario revelan un acercamiento al estudio del hombre en la ejecución del movimiento. La comprensión acerca la motricidad incrementa el interés de los estudiantes-atletas de la escuela de iniciación deportiva escolar y, a su vez, son indispensables en la carrera de Cultura Física, como la estructura interna del cuerpo en el movimiento de articular y muscular.

\footnotetext{
* Artículo de investigación. Cienfuegos, Cuba, Universidad de Cienfuegos "Carlos Rafael Rodríguez", la investigación pertenece al proyecto territorial de desarrollo local titulado "Interacción universidad-sociedad con perspectiva intersectorial del deporte y la salud para el desarrollo del hombre".

** Magíster, Instructora y profesora de Biomecánica, Universidad de Cienfuegos, Cuba. Correo electrónico: atorres@ucf.edu.cu; ORCID: https://orcid.org/0000-0002-0029-7380
} 
Se buscan propuestas didácticas para introducir estos contenidos y así lograr una cultura general integral y formarlos profesionalmente.

Palabras clave: biomecánica, habilidades motrices, mecánica, triatlón, talleres.

\section{The motor skills in triathlon: another look from physics to biomechanics}

\section{Abstract}

This article addresses aspects of motor skills, which are based on the fundamentals of biomechanics, physical and interdisciplinary reasoning. These sciences in the pre-university course reveal an approach to the study of man in the execution of movement. The understanding of motor skills increases the interest of student-athletes in the school of school sports initiation and, in turn, they are indispensable in the Physical Culture career, as the internal structure of the body in joint and muscular movement. Didactic proposals are sought to introduce these contents in order to achieve a comprehensive general culture and to train them professionally.

Keywords: biomechanics, motor skills, mechanics, triathlon, workshops.

\section{Introducción}

La aplicación de la ciencia establece nuevas vías para lograr conocimientos más complejos y la comprensión científica de un mundo sustentado por conceptos, leyes y principios físicos, particularmente en la rama de la mecánica, que se relacionan con diferentes ámbitos de vital importancia en la sociedad, entre ellos el contexto deportivo. Estos conocimientos posibilitan un cambio de pensamiento y conducta en los estudiantesatletas desde la teoría hacia la práctica, y se constituyen en un elemento facilitador en la formación más sólida de una cultura general integral. 
Desde el siglo XIX, se considera en auge el aprendizaje de la educación física desde las ciencias, con énfasis el contexto deportivo, para incrementar la calidad de vida de los atletas. De hecho, la Organización de las Naciones Unidas para la Educación y la Ciencia (UNESCO, 2017) enfatiza en los beneficios del deporte en la salud de los ciudadanos, el cual reduce el riesgo de padecer enfermedades crónicas por el sedentarismo. Malos hábitos como este traen consigo consecuencias negativas, como problemas cardiovasculares y respiratorios. Por otra parte, la misma organización internacional propone actividades de aprendizaje de la educación como objetivos del desarrollo sostenible. Se requiere establecer relaciones coherentes entre el deporte, la salud, la educación y la cultura, pues todas contribuyen el mejoramiento de la sociedad.

En Cuba, las escuelas de iniciación deportiva escolar (EIDE) presentan una educación especial, donde predomina la dualidad de procesos. En esta se debe exigir una correspondencia y un lenguaje común en la docencia y en el entrenamiento deportivo, que se manifiesta en las aptitudes deportivas de los estudiantes-atletas que se destacan desde las primeras edades, entre ellas, las EIDE (Longa, 2015, p. 119).

Ramírez (2018) manifiesta la necesidad del enfoque interdisciplinar entre las ciencias para incrementar el aprendizaje en los estudiantes del preuniversitario. Este criterio es novedoso; sin embargo, deja de manifiesto una carencia del contexto que se estudia. La importancia de relacionar los contextos educacionales a la sociedad es una vía para el desarrollo de las potencialidades, intereses y formación cultural integral de los estudiantes del preuniversitario.

Es significativa la atención que evidencian los autores hacia la cultura general integral de los estudiantes-atletas del preuniversitario. En las EIDE, el tratamiento de los temas debe tener relación con el deporte, de 
modo que se pueda influir en el interés y la comprensión de contenidos que propicien la reflexión y el debate en el aula.

En la encuesta a los estudiantes-atletas se evidenció que las asignaturas de menor preferencia son Física y Matemática, pues manifiestan que es muy abstracto asimilar cada contenido y lo ejemplifican con situaciones de la sociedad. Se identificó que existen en los estudiantes-atletas carencias en el interés y la comprensión de los conocimientos en las asignaturas de ciencias, particularmente en Física. Por consiguiente, las temáticas de mayor interés son las relacionadas con el deporte, que tienen en cuenta el contexto y el perfil ocupacional que elige la mayoría de estos estudiantesatletas después de culminar los estudios en el preuniversitario hacia la carrera universitaria de la Cultura Física.

En las encuestas a los directivos deportivos se evidencia que, en este tipo de escuela deportiva, en particular la EIDE, la principal limitación radica en la exigencia en los deportes de alto rendimiento cuando se busca efectividad de la técnica deportiva. Esto no sucede en la iniciación deportiva, que es la etapa primordial para trabajar las habilidades motrices.

En este mismo sentido, manifiestan que es necesario implementar un enfoque interdisciplinar con las ciencias biológicas aplicadas al deporte, pues su inclusión permite establecer ejercicios especiales dirigidos al perfeccionamiento de la acción motora, con lo cual resultaría más eficaz la ejecución del movimiento.

En general, se evidencian soluciones a problemáticas relacionadas con el perfeccionamiento deportivo; sin embargo, existen insuficiencias en relacionar las experiencias deportivas de los estudiantes-atletas con los conceptos, leyes y principios físicos en la rama de la mecánica, lo que 
incide en la falta de interés por la asignatura y la falta de comprensión acerca la motricidad.

Por lo anterior, es necesario perfeccionar la didáctica de la asignatura de Física relacionándola con los fundamentos biológicos para modificar el interés de los estudiantes por dicha asignatura. De esta forma, se busca lograr mayor comprensión de las habilidades motrices en la ejecución del movimiento. De ahí que el objetivo de la investigación esté encaminado a la elaboración de un sistema de talleres para elevar el interés por la asignatura de Física mediante el estudio de la motricidad en el triatlón desde la biomecánica en las EIDE.

Para lograr tal propósito, se utilizan las ciencias biológicas aplicadas al deporte, donde la biomecánica deportiva desarrolla un rol fundamental, dada su relación con las ciencias ya conocidas desde el preuniversitario. Estudiar la biomecánica deportiva brinda la posibilidad de integrar una cultura general integral en los estudiantes-atletas desde sus experiencias y vivencias deportivas con un enfoque interdisciplinar. Este enfoque muestra su relevancia en el estudio de la motricidad del cuerpo humano dentro de la ejecución de movimientos al momento de estudiar la técnica deportiva.

La comunidad científica internacional actual evidencia un auge considerable desde las ciencias aplicadas al deporte y refiere la necesidad de establecer las relaciones entre de la física, desde la rama de la mecánica, y las ciencias biológicas para la comprensión de las habilidades y capacidades motrices ante el accionar deportivo desde la biomecánica. Por ejemplo, Gómez et ál. (2016) y Estrada (2018), refieren que los fundamentos físicos en la rama de la mecánica pueden ser utilizados para comprender la ejecución de los movimientos en la técnica deportiva, con el empleo de estudios biomecánicos, y ecuaciones físicas y matemáticas. Abordar su estudio implica la necesidad de relacionar varias ciencias con 
un enfoque interdisciplinar para un mismo fin: el entrenamiento deportivo y la calidad de vida de los estudiantes-atletas.

En este mismo sentido, Estrada (2018) asegura que incrementar conocimientos de las ciencias físicas, biologías y químicas acordes es necesario para la eficiencia de la técnica deportiva y evitar el riesgo de lesiones. Todo esto hace posible aumentar el margen de conocimiento integral de los estudiantes-atletas y el perfil ocupacional para su futura profesión hacia la Cultura Física en las EIDE, lo cual es tarea de todos los educadores de la institución. Es meritorio el trabajo en conjunto para elevar los resultados deportivos y seguir sumando atletas a las escuelas deportivas nacionales.

En Cuba también se ha llevado a cabo el perfeccionamiento de la carrera de Cultura Física en la educación superior. La didáctica de las ciencias aplicadas al deporte aporta cada día resultados científicos expresados en tesis de maestrías y doctorados que se orientan a estudiar la ejecución del movimiento y la técnica mediante la biomecánica deportiva en los estudiantes-atletas de alto rendimiento. La base teórica de esta ciencia establece relaciones entre los conceptos, leyes y principios físicos, según Gómez et ál. (2016) y Perdomo (2018).

Cultura Física es una de las carreras de mayor preferencia en los estudiantes-atletas de la EIDE por el conocimiento adquirido desde el accionar deportivo. En esta investigación se hace un análisis de los contenidos que deben adquirir para lograr la mayor comprensión posible con respecto a la ejecución de movimientos mediante la asignatura Biomecánica Deportiva. Para los docentes, es una fortaleza la preferencia deportiva de los estudiantes-atletas, por lo que se debe buscar propuestas didácticas para elevar el interés de la asignatura de Física y su relación con el deporte, de manera planificada, organizada y sistemática, de modo que 
se active el entendimiento de las ciencias biológicas aplicadas al deporte, las cuales constituyen retos del movimiento deportivo cubano.

Longa (2015) enuncia la incorporación de las leyes físicas con situaciones dentro del contexto deportivo en general. Este planteamiento corresponde con lo expresado por el autor Perdomo (2018), quien manifiesta que es necesario que los profesionales de la Cultura Física se fortalezcan desde el conocimiento de las ciencias biológicas aplicadas al deporte para incrementar el rendimiento deportivo en sus atletas. Estos aportes de la ciencia son precisos para esta investigación, pues la propuesta didáctica concebida por el sistema de talleres va dirigida a los estudiantes-atletas, con el fin de que adquieran un mayor entendimiento de la motricidad en el entrenamiento deportivo. Desde esta perspectiva, el propósito es vincular estudios de la estructura interna del atleta cuando ejecuta movimientos, de modo que desarrolle las habilidades motoras específicas del deporte y las capacidades motrices. Este análisis profundiza el trabajo muscular, los ángulos de las articulaciones con un fundamento de leyes, conceptos y principios físicos en el triatlón.

Por su parte, los investigadores Gómez et ál. (2016), Ramírez (2018) y Perdomo (2018) justifican la importancia de incluir los estudios biomecánicos a través de un análisis de las características cinemáticas y dinámicas definidas en la mecánica, la cual se divide en tres grandes aristas: la cinemática, con la que se describe el movimiento de los cuerpos en el espacio sin determinar las causas que la provocan; la cinética, que estudia el gasto energético demandado, y la dinámica, en la cual se profundiza sobre las causas que la originan, se analizan los movimientos articulares y las estructuras musculares ante las cargas aplicadas. En el caso de la cinemática en calidad de sustento de los estudios biomecánicos se valoran conceptos físicos como la rapidez, que determina la distancia recorrida con la unidad de tiempo, por lo cual es una magnitud 
escalar; en ella se desprecia el sentido y dirección del movimiento. Otro concepto similar es la velocidad, una magnitud física vectorial, que se manifiesta en el módulo, sentido y dirección. Esta brinda la posibilitad de que un cuerpo registre la velocidad constante o la varíen.

Desde esta perspectiva conceptual, estos autores plantean que, para el análisis cinemático en la mecánica del movimiento, es importante el sistema de referencia del cuerpo con el espacio y en el tiempo, a través de los cuales se determina el cambio de posición teniendo en cuenta la influencia de agentes externos. Para ello, existen consideraciones en cuanto la masa del cuerpo con proyecciones al eje mecánico que se atribuyen a las magnitudes físicas de longitud, tiempo y masa.

A través de la comprensión de dichos planteamientos, se evidencia que conceptos, leyes y principios físicos comportan un sustento para el movimiento mecánico del cuerpo humano, en el cual se presenta un centro de gravedad, y se manifiestan las palancas y los torques, de acuerdo con los movimientos rotacionales que se aplican en los sistemas osteoarticulares y musculares. Por ejemplo, existe una explicación conceptual y teórica, cuando un segmento del cuerpo se mueve en forma angular desde su articulación (contracción muscular, se produce un torque), en la medición de ángulos entre segmentos adyacentes de la cadena biocinemática, el estado de tensión que se manifiesta con deformación y el aumento de la energía calorífica de las interacciones internas.

De esta forma, el estudiante-atleta logra un dominio de conceptual de la ejecución de movimientos, por medio del estudio de la física, particularmente en la rama de la mecánica. Durante la ejecución de un movimiento se estudian otros contenidos que deben conocer los estudiantes-atletas de triatlón y las disciplinas afines, como las habilidades motrices básicas, relacionando todas las acciones motoras. Ruiz (1997, citado por Cidoncha y Díaz, 2015) manifiestan que, en el triatlón, las 
habilidades básicas que predominan son las locomotrices, al estudiar el movimiento al correr y nadar, y las no locomotrices, al analizar el manejo y dominio del implemento deportivo (la bicicleta) en el espacio.

La edad de la iniciación deportiva en el triatlón está entre los 10 y los 13 años. En esta etapa se inicia el fortalecimiento de las habilidades genéricas comunes y se van especificando las habilidades de cada disciplina para mejorar la ejecución del movimiento. A partir de los 14-17 años, se incluye el desarrollo de habilidades básicas motrices específicas y el trabajo de técnica deportiva en el entrenamiento deportivo.

Ruiz (1997, citado por Cidoncha y Díaz, 2015) relacionan las habilidades motrices específicas con la actividad cognitiva y afectiva-motivacional en el accionar deportivo diario. Estos mediadores son los cognitivos, al propiciar las habilidades intelectuales; los afectivos, al activar el interés del aprendizaje, y el motor, al desarrollar las características de movilidad y su potencial físico y técnico. En su conjunto, permiten el logro de las habilidades motrices específicas en los estudiantes-atletas. Ciertamente, tanto el componente cognitivo como el afectivo deben estar estrechamente relacionados para que sea más efectivo el motor.

En este sentido, antes de realizar cualquier técnica deportiva, los estudiantes-atletas deben tener una previa explicación de cómo debe ser el movimiento; cuál es el de mayor efectividad, y cuál es el funcionamiento mecánico, biológico y químico de las estructuras internas del cuerpo humano. El desarrollo de las habilidades motrices presupone un reto al garantizar los aspectos cognoscitivos y afectivos que integran, de manera que pueda incrementar las capacidades motrices propias del deporte que se estudia. Desde este análisis se deben buscar alternativas desde actividades fuera del aula, donde se integren los conocimientos de docentes y deportivos en las EIDE como hilo conductor del aprendizaje de 
los estudiantes-atletas de estas escuelas deportivas (Perdomo, 2018; Estrada, 2018).

Los estudiantes-atletas que son practicantes de los deportes que pertenecen a tiempo y marca, como el triatlón, deben conocer el funcionamiento del organismo. Para Donskoi y Zatsiorski (1988, citado por Perdomo, 2018) el cuerpo humano constituye un sistema de cadenas biocinemáticas, en cuya esencia prevalece la transmisión del movimiento para lograr su propia ejecución; dicho sistema está conformado por cadenas óseas y uniones biológicas estructurales. Asimismo, las habilidades motrices básicas están presentes en cada acto motor de forma natural, donde existe un gasto energético e influyen los componentes psíquico, fisiológico y técnico en la ejecución del movimiento humano que se desarrolle. Por ello, se hace un análisis biomecánico por disciplinas deportivas. Particularmente, en el triatlón se desarrollan las capacidades motrices de la resistencia, la fuerza, la rapidez y la flexibilidad, por las carreras prolongadas y la trayectoria amplia y compleja de sus transiciones.

Este deporte presenta un enfoque multidisciplinar que vincula las prácticas de natación, ciclismo, carrera y las transiciones. El movimiento de cada disciplina desempeña un papel vital en el rendimiento del estudiante-atleta, donde la técnica en la ejecución deportiva exige el dominio de una serie de habilidades específicas y las capacidades motrices para cada disciplina de este deporte.

Dicha circunstancia implica que una carencia de cualquiera de estas habilidades y capacidades afectará negativamente el rendimiento deportivo. Con esto, no se logra la efectividad de la técnica por consecuencia de los efectos negativos, por lo que provoca deficiencias en el trabajo muscular por falta de conocimiento al respecto. 
La capacidad motriz de la fuerza muscular está dada por la tercera ley de Newton, o ley de acción-reacción, para vencer o contrapesar un peso mediante la actividad muscular. El entrenamiento deportivo busca desarrollar esta cualidad incrementando el dominio técnico y la comprensión del trabajo muscular. Cuando el trabajo muscular es isométrico (o estático) durante la tensión, es el instante donde no varía su longitud, es decir, se fija el movimiento. Cuando el régimen es isotónico (o dinámico), la tensión provoca un cambio de longitud de los músculos. Los trabajos isotónicos concéntricos son aquellos en los que disminuye la longitud, es decir existe la contracción muscular (función agonista), mientras que los excéntricos son aquellos que aumentan la longitud muscular (función antagonista). En este deporte está presente la fuerza. La resistencia, por su parte, es la capacidad de mantener índices elevados de fuerza durante el mayor tiempo posible.

Otro aspecto para tratar dentro de la motricidad es la flexibilidad, en la cual se evidencia componentes morfofuncionales del aparato locomotor que determinan la mayor o menor amplitud de los movimientos del deportista. La carencia de las habilidades motrices limita los niveles de fuerza, velocidad o resistencia. Según Plata (2018), se asume que el desarrollo de la motricidad en los nadadores contribuye al mejoramiento de los problemas cardiovasculares y respiratorios.

En otros trabajos asociados a la calidad de la salud en los atletas, especialmente los ciclistas, se ha investigado la incidencia de la flexibilidad de la articulación coxofemoral y tibiotarsiana sobre el rendimiento de un ciclista en los 20 kilómetros contrarreloj y otro en el kilómetro con partida detenida. En el último caso, se evidencia que la longitud y el tiempo de la ejecución del movimiento son inversamente proporcionales; es decir, gana mayor lentitud, mayor ganancia y a mayor rapidez, mayor será la pérdida. Por ejemplo, para mejorar en un 10 \% las amplitudes de movimiento de la 
articulación coxofemoral, se necesitan entre 40 y 60 sesiones de media hora cada una, mientras que lo adquirido se pierde solo con una semana que no se ejercite.

\section{Metodología}

\section{Grupo de estudio}

La investigación se encuentra entre las estrategias de trabajo de la Facultad de Cultura Física, la cual prioriza la formación de una cultura general integral en los estudiantes-atletas que optan por dicha carrera y consolida las bases de los conocimientos físicos ya adquiridos, los cuales generen nuevos conocimientos biomecánicos.

Desde esta perspectiva, se seleccionó una muestra de 60 estudiantesatletas de los grados décimo y once del preuniversitario de la EIDE “José Agostini Villasana”, en la provincia Cienfuegos durante el curso 218-2019. Se tomó como criterio que fueran practicantes del triatlón y deportes a fines, como atletismo, natación y ciclismo, todos deportes de tiempo y marca. La distribución por deporte fue la siguiente: triatlón (10 atletas), atletismo (20 atletas), natación (20 atletas) y ciclismo (10 atletas). Las edades oscilan entre 15 a 17 años y 20 son del sexo femenino y 40 del sexo masculino.

\section{Instrumentos}

La investigación relaciona los conocimientos físicos y los de fundamentos biológicos, con énfasis los biomecánicos, de manera que se emplearon diferentes métodos teóricos y empíricos. En primer lugar, se implementó el método histórico-lógico, con el fin de sistematizar las concepciones teóricas que existen acerca de la biomecánica deportiva y las características de los deportes de triatlón, el análisis y la síntesis de 
manera que sea posible integrar la información de la bibliografía consultada y los datos recopilados con las técnicas empíricas.

El análisis de documentos permitió analizar los planes de entrenamiento de los deportes seleccionados. Algunos de los analizados fueron los programas de Física décimo grado, programa de Biomecánica Deportiva de la carrera de Cultura Física, informes de los resultados deportivos en el triatlón, resultados de investigaciones referidas al desarrollo de las habilidades y capacidades motrices desde la Biomecánica Deportiva con el fin elaborar un sistema de talleres como propuesta didáctica consolida los contenidos de Física en la rama de la mecánica. El método sistémicoestructural fue utilizado con el fin de elaborar un sistema de talleres. Los métodos empíricos utilizados fueron las entrevistas a los estudiantesatletas que practican el deporte y los deportes afines, así como las encuestas a los directivos deportivos. Con esto se corroboró que la asignatura de Física es una ciencia integradora se relaciona con la biomecánica deportiva y posibilita mejorar los resultados deportivos, al igual que evitar el riesgo de las lesiones. Finalmente, la encuesta a los estudiantes-atletas de la EIDE tuvo como propósito analizar el interés por la asignatura y la comprensión de las habilidades motrices en triatlón.

\section{Enfoque metodológico}

El diseño de investigación asumido se corresponde con un enfoque cualitativo, que permitió analizar la realidad educativa en el contexto de la aplicación de los conocimientos adquiridos en los talleres. En el sistema de talleres, se profundizó en los fundamentos biomecánicos de las habilidades motrices del deporte del triatlón con el empleo de videos para analizar la técnica deportiva y evitar el riesgo de las lesiones. 


\section{Desarrollo de talleres}

La propuesta de talleres está dirigida a las practicantes del deporte triatlón y los deportes afines que estudian en grados décimo y once de la EIDE "Jorge Agostini Villasana” y optan por la carrera de Cultura Física de la Universidad de Cienfuegos en el curso 2018-2019. La idea es que hagan un análisis profundo de los principios, leyes y conceptos físicos que intervienen directamente con los fundamentos biomecánicos. Para su entendimiento, se realizaron cuatro talleres con la participación de sesenta estudiantes-atletas. Ellos presentaron las características generales siguientes:

- Fortalecimiento de los conocimientos ya adquiridos en la asignatura de Física de preuniversitario en la rama de la mecánica.

- $\quad$ Establecer las relaciones entre los principios, leyes y conceptos físicos con los fundamentos biológicos aplicados al deporte, con énfasis en la biomecánica deportiva.

- Análisis de las habilidades motrices en el deporte de triatlón.

- Muestra de resultados de investigaciones realizadas en ese campo de estudio.

- $\quad$ Presencia del intercambio con los entrenadores y directivos deportivos sobre la temática propuesta, ventajas, objetivos propuesta, actividades a desarrollar y contenidos fundamentales.

- Análisis y discusión de las sugerencias para el exitoso desarrollo de las acciones por desarrollar.

Primer taller

Se realizó un intercambio de las mejores prácticas y experiencias en el tratamiento de lesiones más frecuentes. Tuvo como objetivo valorar los fundamentos físicos de la mecánica, sus posibilidades con el uso de videos 
y software Kinovea, así como su importancia en el entrenamiento deportivo del triatlón.

La actividad incita a los alumnos al estudio de la cinemática y dinámica en la traslación y rotación del movimiento, y a las reflexiones acerca de la importancia y actualidad de su aplicación en la práctica cotidiana de este deporte por ser un deporte olímpico. Además, el taller enfatiza en la observación de videos y el conocimiento del software en los estudios biomecánicos, así como en la posibilidad de revisión de artículos en internet.

\section{Segundo taller}

Se realizó un intercambio de las mejores prácticas y experiencias en el tratamiento de las ciencias físicas, biológicas y químicas desde la biomecánica deportiva, que tuvo como objetivo valorar el tratamiento de los contenidos con enfoque disciplinario y su aplicación en el entrenamiento deportivo de triatlón.

Las temáticas de la enseñanza del taller fueron cuatro, a saber: el surgimiento, actualidad y perspectivas de la biomecánica deportiva; el hombre como sistema biomecánico sustentado por conceptos, leyes y principios físicos; interdisciplinariedad y valor de los métodos biomecánicos de investigación. Las habilidades motrices de las transiciones del deporte triatlón.

Tercer taller

Se trabajó sobre las características biomecánicas que se manifiestan en los movimientos humanos durante la actividad deportiva y uso de las TIC para su análisis. Este taller tuvo como objetivo caracterizar los movimientos de traslación y rotación, sus características, ecuaciones y gráficas, así como el procedimiento para el análisis biomecánico de los movimientos, teniendo 
en cuenta la aplicación del software en el análisis de ejecuciones técnicas de la práctica deportiva.

Los contenidos tratados fueron: las características cinemáticas del movimiento, ecuaciones y gráficos; los movimientos de traslación y rotación; el uso y ventajas del software para el análisis de los movimientos deportivos, con el cual se le da tratamiento a las técnicas; los conocimientos biomecánicos que dependen de los principios, leyes y conceptos de la mecánica que rigen la motricidad humana y la biomecánica como ciencia; métodos de trabajo; técnicas de investigación biomecánica y su aplicación para la obtención de características cinemáticas y dinámicas del movimiento humano.

\section{Cuarto taller}

Este taller se ocupó de estudiar las capacidades motrices en el triatlón por la observación de videos de atletas con maestría deportiva en este deporte, así como el uso del software y su factibilidad en el análisis de los movimientos humanos. El objetivo del taller fue aplicar el software informático a los métodos y técnicas de carácter biomecánicos que posibilitan estudiar del movimiento humano e indicadores propios de su técnica deportiva.

Los contenidos abordados fueron: caracterización del proceso de filmación; movimientos en los ejes y los planos; ejemplificación del software Kinovea, utilizado en el análisis biomecánico de los movimientos humanos, y determinación de indicadores, a partir de la aplicación del análisis de videos en acciones motoras con el objetivo de analizar las características cinemáticas y dinámicas del movimiento humano y las habilidades motrices. 


\section{Resultados y discusión}

Con la aplicación del software Kinovea y el análisis de videos deportivos, se pudo profundizar en la ejecución de los movimientos de los atletas en este tipo de deporte, en la determinación de variables como: trayectoria, postura, centro de masa, centro de gravedad, aceleración, rapidez, ángulos, fuerza, fases, entre otras).

Se logró mayor interés por los conocimientos físicos en la rama de la mecánica, en la medida en que se desarrolló en los estudiantes-atletas una cultura general integral. Se logró la incorporación de practicantes de atletismo, natación y ciclismo que desean conocer sobre el deporte de triatlón. Por esto, se evidenció gran impacto en el mejoramiento de las técnicas de transición motora.

Tabla 1. Resultados de la encuesta "Interés por la física en la rama de la mecánica y la comprensión acerca la motricidad desde la biomecánica" (antes y después)

\begin{tabular}{|c|c|c|c|c|}
\hline Talleres & Antes & $\%$ & Después & $\%$ \\
\hline Primer taller & 25 & 41 & 37 & 61 \\
\hline Segundo taller & 38 & 63 & 42 & 70 \\
\hline Tercer taller & 46 & 76 & 54 & 90 \\
\hline Cuarto taller & 55 & 91 & 60 & 100 \\
\hline
\end{tabular}

Fuente: elaboración propia

Tabla 2. Resultados deportivos en el triatlón en la EIDE de la provincia de Cienfuegos desde los cursos 2017-2018 al 2018-2019

\begin{tabular}{|c|c|c|}
\hline Categoría & Curso 2017-2018 & Curso 2018-2019 \\
\hline Categoría escolar 13-15 & $7 .^{\circ}$ & $6 .^{\circ}$ \\
\hline Categoría juvenil 16-20 & $5 .^{\circ}$ & $3 .^{\circ}$ \\
\hline
\end{tabular}

Fuente: elaboración propia. 
Con la puesta en práctica de la propuesta sistema de talleres, se hizo un análisis cualitativo de las habilidades y capacidades motrices desde la biomecánica en el triatlón fundamentado con conceptos, leyes y principios físicos, al punto de implementar la interdisciplinariedad entre las ciencias biológicas y el deporte. Se logró un mayor interés por la asignatura de Física, incremento y profundización en el aprendizaje, y mayor comprensión por parte de los estudiantes-atletas acerca la motricidad. Esto tuvo gran impacto en el mejoramiento de las técnicas de transición motora y resultados deportivos considerables en ambas categorías. Se obtuvieron 50 (83 \%) de medallas entre el deporte de triatlón y los deportes a fin seleccionados, y 5 (50\%) de los estudiantes-atletas que obtuvieron medallas en el triatlón fueron seleccionados como perspectivas para el equipo nacional.

\section{Aplicación de la técnica "Positivo, negativo e interesante"}

Al culminar los talleres se realizó la técnica "Positivo, negativo e interesante", con el fin de constatar los nuevos conocimientos biomecánicos en los practicantes en el deporte de triatlón. Mediante esta se emitieron los criterios sobre el interés de las estudiantes-atletas por la física relacionada con las ciencias biológicas aplicadas al deporte y los que corresponden a cada categoría. Se observa una modificación considerable de los resultados obtenidos de la encuesta de los estudiantes-atletas, pues estos manifestaron un alto interés por los talleres y el conocimiento que adquirieron en ellos. Además, emitieron sus criterios en las categorías de lo positivo, negativo e interesante que se presentan a continuación.

Positivo:

- Importancia de la física mecánica para la sociedad, especialmente el deporte. 
- La necesidad de su aplicación con otras ciencias para estudiar el movimiento mecánico del hombre y la motricidad en la actividad física deportiva desde la biomecánica.

- La importancia de los softwares para hacer análisis de la técnica y el mejoramiento deportivos.

- El impacto de los biomecánicos y la necesidad de revolucionar las formas de realizar los estudios biomecánicos

Negativo:

- Debe ser más tiempo por la complejidad de los conocimientos.

- No hay nada que no me gustara.

- Todo me gustó, pero hubo carencia de medios y tecnologías para su mayor y cotidiana realización.

Interesante:

- Me sorprendí al estudiar mi cuerpo cuando hago algún movimiento en mi deporte. Estudiarlo desde las ciencias biológicas.

- Es novedoso la aplicación del software Kinovea para el estudio del deporte tan complejo que es el triatlón.

- El impacto que tiene la biomecánica deportiva desde entrenamiento deportivo.

\section{Conclusiones}

Con la investigación se determinaron los fundamentos físicos con enfoque interdisciplinar que sustentan la problemática del interés de los estudiantes-atletas por la asignatura de Física, en relación con las ciencias biológicas aplicadas al deporte. Se evidenció una mayor comprensión acerca de la motricidad desde la biomecánica en los estudiantes-atleta de las EIDE. 
Con el empleo del sistema de talleres se logra un mejoramiento en la técnica y resultados deportivos en competencias. Los videos de diferentes atletas de triatlón a nivel internacional y nacional, así como estudios biomecánicos de las habilidades específicas y capacidades motrices del deporte, conducen a un aprendizaje complejo y significativo para los estudiantes-atleta.

\section{Referencias}

Cidoncha, V. y Díaz, E. (2015). Aprendizaje motor. Las habilidades motrices básicas: coordinación y equilibrio. Revista EFDeportes.com, 15(147). https://www.efdeportes.com/efd147/habilidades-motrices-basicas-coordinaciony-equilibrio.htm

Estrada, Y. (2018). Biomecánica: de la física mecánica al análisis de gestos deportivos. Ediciones USTA. http://repository.usta.edu.co/handle/11634/12464

Gómez, A. P., Ramírez, S., Infante, N. M., Pavón, J. L. y Pozo, R. J. (2016). La biomecánica al servicio de la actividad física en Holguín. Deporvida, 16(42), 89102. https://deporvida.uho.edu.cu/index.php/deporvida/article/view/569

Longa, T. M. (2015). El aprendizaje significativo de la Física para estudiantes-atletas de las escuelas deportivas Rastros Rostros, 17(31).

https://doi.org/10.16925/ra.v17i31.1093

Plata, D. (2018). Mejora del desarrollo motor a través de la natación en el alumnado de Educación Primaria. Efdeportes, 23(247).

Perdomo, J. (2018). La superación profesional de entrenadores deportivos [tesis doctoral, Universidad Central "Marta Abreu"]. Repositorio institucional UCLV. https://dspace.uclv.edu.cu/handle/123456789/11252

Ramírez, Y. (2018). La Preparación de los profesores de Física en preuniversitario para la interdisciplinariedad en el proceso de enseñanza-aprendizaje [tesis doctoral]. Universidad Central "Marta Abreu”. Villa Clara, Cuba. 